Mon. Not. R. Astron. Soc. 000, 000-000 (0000) Printed 19 February $2019 \quad$ (MN LATEX style file v2.2)

\title{
A new fast radio burst in the datasets containing the Lorimer burst
}

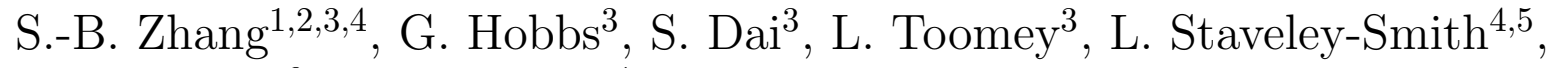 \\ C. J. Russell ${ }^{6}$ and X.-F. $\mathrm{Wu}^{1}$ \\ ${ }^{1}$ Purple Mountain Observatory, Chinese Academy of Sciences, Nanjing 210008, China \\ ${ }^{2}$ University of Chinese Academy of Sciences, Beijing 100049, China \\ ${ }^{3}$ CSIRO Astronomy and Space Science, Australia Telescope National Facility, Box 76, Epping, NSW 1710, Australia \\ ${ }^{4}$ International Centre for Radio Astronomy Research, University of Western Australia, Crawley, WA 6009, Australia \\ ${ }^{5}$ ARC Centre of Excellence for All Sky Astrophysics in 3 Dimensions (ASTRO 3D) \\ ${ }^{6}$ CSIRO Scientific Computing Services, Australian Technology Park, Locked Bag 9013, Alexandria, NSW 1435, Australia
}

19 February 2019

\begin{abstract}
We report the discovery of a new fast radio burst (FRB), FRB 010312, in archival data from a $1.4 \mathrm{GHz}$ survey of the Magellanic Clouds using the multibeam receiver on the Parkes $64 \mathrm{~m}$-diameter radio telescope. These data sets include the Lorimer burst (FRB 010724), which it pre-dates and which we also re-detect. The new burst has a much higher dispersion measure of $1187 \mathrm{~cm}^{-3} \mathrm{pc}$. The burst is one of the broadest found to date, the second earliest FRB known, and the ninth FRB discovered with a dispersion measure larger than $1000 \mathrm{~cm}^{-3} \mathrm{pc}$. Our discovery indicates that there are likely to be more burst events still to be found in the existing Parkes data archive.
\end{abstract}

Key words: Radio continuum: transients - methods: data analysis

\section{INTRODUCTION}

The first reported fast radio burst (FRB) was detected as a bright single pulse of millisecond duration using the Parkes $64 \mathrm{~m}$-diameter radio telescope (Lorimer et al. 2007). The FRB was discovered by reprocessing archival observations that had originally been observed in order to search for pulsars in the Magellanic clouds (Manchester et al. 2006). Those observations are available in the ATNF pulsar data archive (Hobbs et al. 2011) and, in this paper, we report the discovery of a second FRB in the same data set.

Since the initial discovery, more than 60 FRBs have been published ${ }^{1}$ (Petroff et al. 2016) and their high dispersion measure (DM), in excess of the expected contribution from the Milky Way, implies an extragalactic origin. Furthermore, the localization of one repeating FRB to a dwarf galaxy at redshift $z \sim 0.2$ (Spitler et al. 2016, Chatterjee et al. 2017. Tendulkar et al. 2017), is consistent with the DM-estimated distance and, assuming that the repeating FRBs are similar in origin to those discovered by the Parkes telescope, confirms that FRB progenitors are at cosmic distances. The most successful telescopes for finding FRBs have been Parkes (Lorimer et al. 2007; Keane et al. 2012, Thorn- ton et al. 2013, Burke-Spolaor \& Bannister 2014, Ravi, Shannon, \& Jameson 2015, Petroff et al. 2015 Keane et al. 2016. Champion et al. 2016, Ravi et al. |2016 Petroff et al.| 2017. Bhandari et al. 2018, Petroff et al. 2019), Molonglo (Caleb et al. 2017), ASKAP (Shannon et al. 2018; Macquart et al. 2018) and, most recently, CHIME (The CHIME/FRB Collaboration 2019a b). The distribution of the DMs for the detected FRB population seems to vary between telescopes. This is explained by a combination of the telescope sensitivity and field-of-view with various models of FRB event rates (Shannon et al. 2018). In particular, the four FRBs with the highest DMs were discovered with the Parkes telescope (which, including the one presented here, has found seven out of the nine known FRBs with a DM larger than $\left.1000 \mathrm{~cm}^{-3} \mathrm{pc}\right)$.

The progenitors of FRBs remain mysterious, although plenty of models have been proposed. According to their short duration $(\sim \mathrm{ms})$ and extremely high inferred brightness temperature $\left(\gtrsim 10^{35} \mathrm{~K}\right)$, a coherent emission process (Melrose \& Yuen 2016) has been invoked to explain the radiation. Such emission processes include magnetic reconnections of a neutron star's magnetosphere caused by the outflow from a super-massive black hole in the host galaxy (Zhang 2018) and supergiant pulses from extragalactic pulsars (Cordes \& Wasserman 2016). Other models involve merging neutron stars (Totani 2013, Wang et al. 2016) 
or white dwarf stars (Kashiyama, Ioka, \& Mészáros 2013), collapse of super-massive neutron stars to black holes (Falcke \& Rezzolla 2014), magnetar flares (Popov \& Postnov 2013), neutron star-white dwarf binary accretion (Gu et al. 2016) and even cosmic string collisions (Cai et al. 2012), collisions between neutron stars and asteroids (Huang \& Geng 2016 Dai et al. 2016) or charged black hole mergers (Zhang 2016 Liu et al. 2016, Deng et al. 2018). However, most current models cannot yet be confirmed, nor ruled out, because of the insufficient sample of FRBs.

In this letter, we present a new FRB (known as FRB 010312) detected during reprocessing of archival data from a $1.4 \mathrm{GHz}$ survey of the Magellanic Clouds. This FRB is the second earliest FRB yet detected, one of the widest, and the ninth FRB discovered with DM larger than $1000 \mathrm{~cm}^{-3}$ pc. In Section 2, we describe the details of the observations and data reduction. The properties of the burst and discussion of our detection are presented in Section 3 We conclude in Section 4

\section{OBSERVATION AND DATA REDUCTION}

Manchester et al. (2006) carried out the survey of the large and small Magellanic Clouds with the primary goal of discovering pulsars. The survey was undertaken with the Parkes $64 \mathrm{~m}$-diameter radio telescope between May 2000 to $\mathrm{Au}-$ gust 2001 and led to the discovery of 14 pulsars. Other known pulsars were redetected and the survey observations also included observations of bright known pulsars, such as PSR J0437-4715, in order to confirm that the system was working correctly. The observations for this survey have been archived in the Parkes data archive (https: //data.csiro.au see Hobbs et al. (2011) for details) using the project code identifier P269. The survey used the $21 \mathrm{~cm}$ multibeam receiver centred at $1374 \mathrm{MHz}$. The channelised and polarization-summed signals were one-bit sampled and recorded using an Analogue Filter Banks (AFB) system. The bandwidth, number of channels and sampling time of these observations are $288 \mathrm{MHz}, 96$ and $1 \mathrm{~ms}$, respectively.

Lorimer et al. (2007) searched for bright single pulses up to a DM of $500 \mathrm{~cm}^{-3} \mathrm{pc}$ and successfully identified a burst with a DM of $375 \mathrm{~cm}^{-3} \mathrm{pc}$, which is now known to be the first detection of an FRB. There are no published searches of the same data set to higher DMs. We are carrying out a project to reprocess all the search-mode observations in the data archive to search for events such as FRBs. We started with the Magellanic Cloud survey primarily to confirm that we can re-detect the Lorimer et al. (2007) event. The survey data contains approximately $6250 \mathrm{~h}$ of on-sky integration time. This corresponds to $\sim 267 \mathrm{deg}^{2} \mathrm{~h}$. A recent Parkes survey with the same receiver system (the HTRU survey) had an FRB event rate of $1 / 144 \mathrm{deg}^{-2} \mathrm{~h}^{-1}$ (Champion et al. 2016). We therefore predicted that the Magellanic Cloud survey data should include around two FRBs.

We used the pulsar searching software package PRESTq ${ }^{2}$ (Ransom 2001) and processed the data on CSIRO's high performance computer facilities. Strong narrow-band and short-duration broadband radio frequency interference

2 http://www.cv.nrao.edu/ sransom/presto/
Table 1. The properties of FRB 010312.

\begin{tabular}{|c|c|}
\hline \multicolumn{2}{|l|}{ Observed Properties } \\
\hline Event data UTC & 2001 March 12 \\
\hline Event time UTC, $\nu_{1} .374 \mathrm{GHz}$ & $11: 06: 47.98$ \\
\hline Event time Local (AEDT), $\nu_{1} .374 \mathrm{GHz}$ & $22: 06: 47.98$ \\
\hline Pointing R.A. (J2000) & $05: 26: 54.9$ \\
\hline Pointing Dec. (J2000) & $-64: 56: 19.2$ \\
\hline Galactic longitude & $274.72^{\circ}$ \\
\hline Galactic latitude & $-33.30^{\circ}$ \\
\hline Beam 7 full-width, half-maximum & $14.1^{\prime}$ \\
\hline $\mathrm{DM}\left(\mathrm{cm}^{-3} \mathrm{pc}\right)$ & $1187 \pm 14$ \\
\hline Observed width (ms) & $24.3 \pm 1.3$ \\
\hline $\mathrm{S} / \mathrm{N}$ & 11 \\
\hline \multicolumn{2}{|l|}{ Inferred Properties } \\
\hline Peak flux density (Jy) & 0.25 \\
\hline Fluence (Jy ms) & 6.1 \\
\hline $\mathrm{DM}_{\mathrm{MW}, \mathrm{NE} 2001}\left(\mathrm{~cm}^{-3} \mathrm{pc}\right)$ & 51 \\
\hline $\mathrm{DM}_{\mathrm{MW}, \mathrm{YMW} 16}\left(\mathrm{~cm}^{-3} \mathrm{pc}\right)$ & 55 \\
\hline $\mathrm{DM}_{\mathrm{MC}, \mathrm{YMW} 16}\left(\mathrm{~cm}^{-3} \mathrm{pc}\right)$ & 12 \\
\hline Redshift $_{\mathrm{YMW} 16}, \mathrm{z}$ & $1.4^{\mathrm{a}}$ \\
\hline DistanceYMW16 (Gpc) & $3.9^{\mathrm{a}}$ \\
\hline
\end{tabular}

(RFI) were identified and marked using the PRESTO routine RFIFIND. We used a $1 \mathrm{~s}$ integration time for our Radio Frequency Interference (RFI) identification and the default cutoff to reject time-domain and frequency-domain interference in our pipeline. To avoid deleting possible bursts, we used the option NOCLIP during all the processing. In preparation for de-dispersion, the DDPLAN.PY algorithm was used to determine the DMs required for us to search. The DM range that we searched was 0 to $5000 \mathrm{~cm}^{-3}$ pc and the number of DM trials was 440. Data were then de-dispersed at each of the trial DMs using the PREPDATA routine, and RFI was removed based on the mask file. Single pulse candidates with a signal-to-noise ratio $(\mathrm{S} / \mathrm{N})$ larger than five were identified using the SINGLE_PULSE_SEARCH.PY routine for each dedispersed time series ${ }^{3}$ All of the several tens of thousands of candidates were ranked and plotted using the same method as Zhang et al. (2018). Those that were not clearly caused by RFI were visually inspected.

\section{RESULTS AND DISCUSSION}

In our processing, we re-detected the original FRB in the observation from 2001 July 24 at a DM of $375 \mathrm{~cm}^{-3} \mathrm{pc}$, in three beams (beams 6, 7 and 13) with $\mathrm{S} / \mathrm{N}$ of 31,16 and 26 respectively. We therefore are confident in the quality of the archived data, our processing, visualisation and ranking methods.

A new FRB with a DM of $1187 \pm 14 \mathrm{~cm}^{-3}$ pc and

3 We used the command-line option $-\mathrm{b}$ and boxcar filtering with filter widths of $1,2,3,4,6,9,14,20,30,45,70,100,150,220$ and 300 sample. The maximum search was equivalent to a width of $300 \mathrm{~ms}$. We use the definition of $\sigma$ as presented by PRESTO for our $\mathrm{S} / \mathrm{N}$ value. 
$\mathrm{S} / \mathrm{N}=11$ was also detected in our search. Figure 1 shows the burst in the frequency-time plane and its integrated pulse profile after being de-dispersed at the optimal DM value. We can see that the signal is much stronger in the lower part of the observing band. This is similar to FRBs such as FRB 110214 (Petroff et al. 2019) and FRB 171019 (Shannon et al. 2018). There are also two strong narrow-band RFI signatures close to the band edges, but they do not affect our ability to detect the FRB.

The properties of FRB 010312 are presented in Table 1 Our FRB ${ }^{4}$ was detected in only a single beam (Beam 7 ) of the multibeam receiver. The remaining 12 beams show no clear candidate, nor RFI around the time of the burst. We therefore cannot precisely provide the position of the source. In Table 1 we simply list the pointing position of the beam in right ascension and declination (and converted to Galactic coordinates).

The burst has a width of $24.3 \pm 1.3 \mathrm{~ms}$ at its $50 \%$ power point, which is one of the largest values reported thus far (note that Farah et al. (2017) report an FRB with a width of $26 \mathrm{~ms}$ ). We have searched for changes in the pulse width as a function of observing frequency, but the $\mathrm{S} / \mathrm{N}$ of our profile precludes any detailed analysis.

From the estimated S/N, DM and sky position we can infer various properties that are listed in the lower half of Table 1. The peak flux density was obtained from the single pulse radiometer equation (Cordes \& McLaughlin 2003) and the S/N measurement. The YMW16 electron density model (Yao, Manchester, \& Wang 2017), which assumes $H_{0}=67.3 \mathrm{~km} \mathrm{~s}^{-1} \mathrm{Mpc}^{-1}$ (The Planck Collaboration 2014) and the local intergalactic medium baryon density $n_{\mathrm{IGM}}=0.16 \mathrm{~m}^{-3}$ (Katz 2016), indicates a large cosmic distance of $3.9 \mathrm{Gpc}$ with the assumption of the host galaxy DM $\sim 100 \mathrm{~cm}^{-3} \mathrm{pc}$.

The burst was only detected in one beam and in order to check whether there is any evidence of repeated bursts from this FRB, we used $46.2 \mathrm{~h}$ of archival observations whose pointing positions were within $0.5 \mathrm{deg}$ of the position of the beam in which FRB 010312 was detected. We searched a DM range from 1158 to $1178 \mathrm{~cm}^{-3}$ pc with a DM step of $0.1 \mathrm{~cm}^{-3} \mathrm{pc}$, but no new convincing candidates were detected.

The results from processing the entire $6250 \mathrm{~h}-$ long survey led to the detection of the original FRB, the new one as described above, and the expected single pulse detections from known pulsars. Foster et al. (2018) presented a set of tests that can be applied to give confidence to any potential FRB discovery. We have applied these tests to both FRB 010312 and the Lorimer burst. We obtain almost identical test results providing confidence that our new FRB is real. However, FRB 010312 is not bright enough to enable a detailed study of the spectral and scattering properties, although in common with many other FRBs, FRB 010312 is significantly weaker in part of the band. We found no further FRB candidate with $\mathrm{S} / \mathrm{N} \geqslant 8$ although around a dozen candidates with $\mathrm{S} / \mathrm{N} \geqslant 7$ were detected. We will discuss these

\footnotetext{
${ }^{4}$ Since the discovery of the first FRB searches have been undertaken in even earlier data sets. The earliest FRB yet detected is FRB 010125 again detected in archival Parkes data and our new FRB (FRB 010312) is therefore the second-earliest known FRB.
}

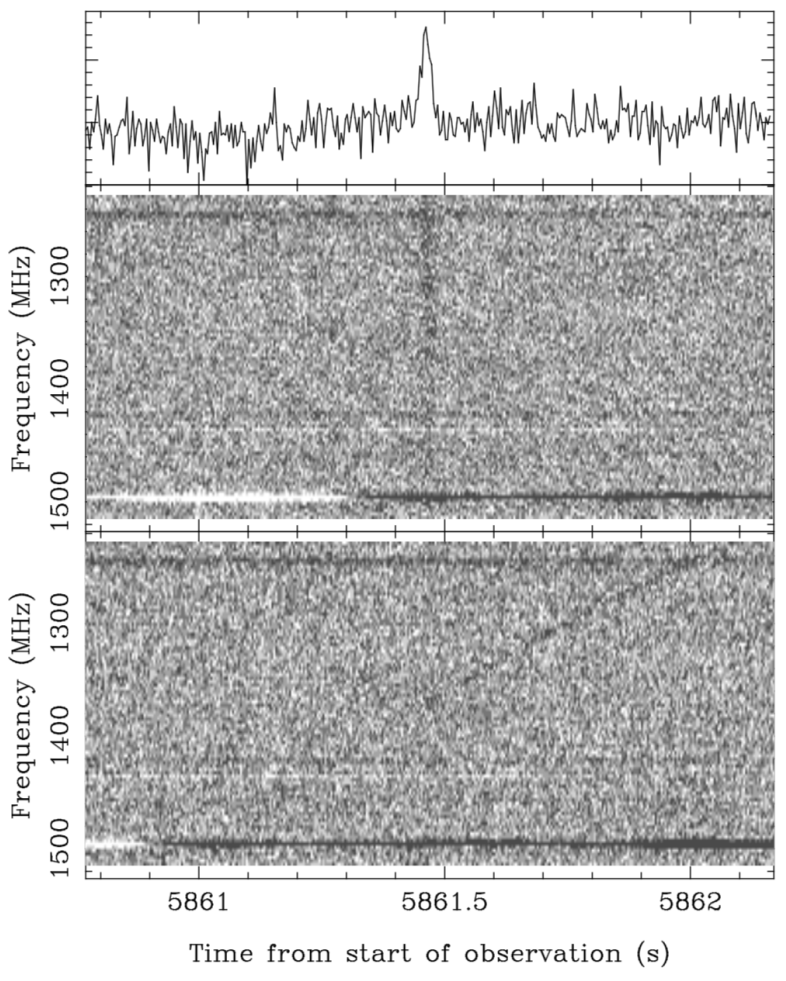

Figure 1. Frequency-time plane of the FRB 010312 without dispersion (bottom), with dispersion (central) and its integrated pulse profile (shown using an arbitrary flux scale) after being de-dispersed at the optimal DM value (top). The frequency resolution is $3 \mathrm{MHz}$. The time axis shows the time since the start of the observation (March 12, 2001 09:29:07 UTC) with a resolution of $4 \mathrm{~ms}$.

much weaker signals further when we have completed our larger-scale analysis of the entire data archive.

With the addition of FRB 010312, the FRB event rate of the Magellanic Clouds survey is $1 / 134 \mathrm{deg}^{-2} \mathrm{~h}^{-1}$, which is similar to the prediction of Champion et al. (2016) of $1 / 144 \mathrm{deg}^{-2} \mathrm{~h}^{-1}$ from the Parkes HTRU survey, which was based on 10 FRB detections. However, Petroff et al. (2019) has recently found a further FRB in this same data set giving an updating event rate closer to our measurement of $1 / 131 \mathrm{deg}^{-2} \mathrm{~h}^{-1}$.

\section{CONCLUSION}

Since Lorimer et al. (2007) discovered the first FRB, new bursts continue to be discovered using archival data with different DM trials and new search algorithms (e.g., BurkeSpolaor \& Bannister 2014, Champion et al. 2016, Petroff et al. 2019). There are many reasons why FRBs were missed in some searches, including the challenges of looking through large numbers of candidates and different methods for dealing with RFI. However, the most significant issue is simply the range of DMs searched. Searching over a very large DM range is computationally expensive and can increase the false alarm rate, but, as presented here, can lead to new discoveries.

We have reported here on a single FRB that occurred 
Zhang et al.

many years ago with a relatively simple observing system. We therefore cannot do detailed follow-up, multi-wavelength observations, nor carry out an in-depth analysis of the scattering, scintillation or polarisation properties of the burst. However, our results do provide confidence in the event rate predictions. The recent predictions indicated that two FRBs should be detectable in the Magellanic Cloud survey and so our results confirm that these predictions are reliable.

Parkes is one of the most sensitive telescopes used to find FRBs and so can find relatively weak bursts (when compared, for example, to the ASKAP discoveries). The FRB reported here is one of only a handful of FRBs with DMs higher than $1000 \mathrm{~cm}^{-3}$ pc and has one of the largest isotropic energy of $4.3 \times 10^{33} \sqrt{5}$ As we continue to search the Parkes archival data we expect to increase the sample size for these extremely high DM FRB events. Telescopes such as ASKAP and CHIME are now finding large numbers of FRBs (Shannon et al. 2018; The CHIME/FRB Collaboration 2019a). However, the Parkes multibeam data sets provide an enormous repository of data obtained with a single telescope and receiver system. Even though the data are all in a similar format, different research groups have processed their data sets in different ways. With the archive and supercomputing facilities available we now, for the first time, have the opportunity of processing all the data in a self-consistent manner. We are confident that we will discover new FRBs in this processing and obtain a reliable FRB event rate over decades of observations.

\section{ACKNOWLEDGMENTS}

The Parkes radio telescope is part of the Australia Telescope National Facility which is funded by the Australian Government for operation as a National Facility managed by CSIRO. This paper includes archived data obtained through the CSIRO Data Access Portal (http://data.csiro.au). This work was supported by a China Scholarship Council (CSC) Joint PhD Training Program grant and the National Natural Science Foundation of China (Grant No. 11725314). Parts of this research were supported by the Australian Research Council Centre of Excellence for All Sky Astrophysics in 3 Dimensions (ASTRO 3D), through project number CE170100013.

\section{REFERENCES}

Bhandari S., et al., 2018, MNRAS, 475, 1427

Burke-Spolaor S., Bannister K. W., 2014, ApJ, 792, 19

Cai Y.-F., Sabancilar E., Steer D. A., Vachaspati T., 2012, PhRvD, 86, 043521

Caleb M., et al., 2017, MNRAS, 468, 3746

Champion D. J., et al., 2016, MNRAS, 460, L30

Chatterjee S., et al., 2017, Natur, 541, 58

Cordes J. M., McLaughlin M. A., 2003, ApJ, 596, 1142

Cordes J. M., Wasserman I., 2016, MNRAS, 457, 232

${ }^{5}$ It is estimated using the definition in Petroff et al. (2016) with adopting $H_{0}=67.3 \mathrm{~km} \mathrm{~s}^{-1} \mathrm{Mpc}^{-1}, \Omega_{\mathrm{m}}=0.315$ and $\Omega_{\Lambda}=$ 0.685 (The Planck Collaboration 2014)
Dai Z. G., Wang J. S., Wu X. F., Huang Y. F., 2016, ApJ, 829, 27

Deng C.-M., Cai Y., Wu X.-F., Liang E.-W., 2018, PhRvD, 98, 123016

Falcke H., Rezzolla L., 2014, A\&A, 562, A137

Farah W., et al., 2017, ATel1, 10867,

Foster G., et al., 2018, MNRAS, 481, 2612

Gu W.-M., Dong Y.-Z., Liu T., Ma R., Wang J., 2016, ApJ, 823, L28

Hobbs G., et al., 2011, PASA, 28, 202

Huang Y. F., Geng J. J., 2016, ASPC, 502, 1

Kashiyama K., Ioka K., Mészáros P., 2013, ApJ, 776, L39

Katz J. I., 2016, ApJ, 818, 19

Keane E. F., Stappers B. W., Kramer M., Lyne A. G., 2012, MNRAS, 425, L71

Keane E. F., et al., 2016, Natur, 530, 453

Liu T., Romero G. E., Liu M.-L., Li A., 2016, ApJ, 826, 82

Lorimer D. R., Bailes M., McLaughlin M. A., Narkevic

D. J., Crawford F., 2007, Sci, 318, 777

Macquart J.-P., Shannon R. M., Bannister K. W., James C. W., Ekers R. D., Bunton J. D., 2018, arXiv, arXiv:1810.04353

Manchester R. N., Fan G., Lyne A. G., Kaspi V. M., Crawford F., 2006, ApJ, 649, 235

Melrose D. B., Yuen R., 2016, JPlPh, 82, 635820202

Petroff E., et al., 2015, MNRAS, 447, 246

Petroff E., et al., 2016, PASA, 33, e045

Petroff E., et al., 2017, MNRAS, 469, 4465

Petroff E., et al., 2019, MNRAS, 482, 3109

Popov S. B., Postnov K. A., 2013, arXiv, arXiv:1307.4924

Ransom S. M., 2001, PhDT,

Ravi V., et al., 2016, Sci, 354, 1249

Ravi V., Shannon R. M., Jameson A., 2015, ApJ, 799, L5

Shannon R. M., et al., 2018, Natur, 562, 386

Spitler L. G., et al., 2016, Natur, 531, 202

Tendulkar S. P., et al., 2017, ApJ, 834, L7

The CHIME/FRB Collaboration, 2019a, arXiv, arXiv:1901.04524

The CHIME/FRB Collaboration, 2019b, arXiv, arXiv:1901.04525

The Planck Collaboration, 2014, A\&A, 571, A16

Thornton D., et al., 2013, Sci, 341, 53

Totani T., 2013, PASJ, 65, L12

Wang J.-S., Yang Y.-P., Wu X.-F., Dai Z.-G., Wang F.-Y., 2016, ApJ, 822, L7

Yao J. M., Manchester R. N., Wang N., 2017, ApJ, 835, 29

Zhang B., 2016, ApJ, 827, L31

Zhang B., 2018, ApJ, 854, L21

Zhang S.-B., Dai S., Hobbs G., Staveley-Smith L., Manchester R. N., Russell C. J., Zanardo G., Wu X.-F., 2018, MNRAS, 479, 1836 